\title{
Editorial: Advances and Current Challenges in Calcium Signaling Within the Cardiovascular System
}

\author{
Roberto Berra-Romani ${ }^{1 *}$, Germano Guerra ${ }^{2 *}$ and Francesco Moccia ${ }^{3 *}$ \\ ${ }^{1}$ Department of Biomedicine, School of Medicine, Benemérita Universidad Autónoma de Puebla, Puebla, Mexico, \\ ${ }^{2}$ Department of Medicine and Health Sciences "V. Tiberio", University of Molise, Campobasso, Italy, ${ }^{3}$ Laboratory of General \\ Physiology, Department of Biology and Biotechnology "L. Spallanzani”, University of Pavia, Pavia, Italy
}

Keywords: excitation-contracting coupling, endothelial signaling, heart failure, cardiac arrhtyhmias, phenotypic swich, angiogenesis, vascular permeability

\section{Editorial on the Research Topic}

\section{Advances and Current Challenges in Calcium Signaling Within the Cardiovascular System}

Calcium signaling in the cardiovascular system does not only drive "excitation-contraction" coupling in cardiomyocytes and vascular smooth muscle cells (VSMCs) (Eisner et al., 2017). Intracellular $\mathrm{Ca}^{2+}$ signals finely tune endothelial functions, thereby regulating also vascular tone and permeability, angiogenesis and vasculogenesis, coagulation and inflammation (Smani et al.,

OPEN ACCESS

Edited and reviewed by: Eduardo Nava,

University of Castilla-La

Mancha, Spain

*Correspondence:

Francesco Moccia

francesco.moccia@unipv.it

Roberto Berra-Romani

rberra001@hotmail.com

Germano Guerra

germano.guerra@unimol.it

Specialty section:

This article was submitted to

Vascular Physiology,

a section of the journal

Frontiers in Physiology

Received: 16 April 2021 Accepted: 21 May 2021

Published: 23 July 2021

Citation:

Berra-Romani R, Guerra G and Moccia F (2021) Editorial: Advances and Current Challenges in Calcium Signaling Within the Cardiovascular System. Front. Physiol. 12:696315. doi: 10.3389/fphys.2021.696315 2018). An increase in intracellular $\mathrm{Ca}^{2+}$ concentration $\left(\left[\mathrm{Ca}^{2+}\right]_{i}\right)$ can be achieved through the concerted interaction among the components of a versatile network of membrane receptors, ion channels, transporters and buffers that can be uniquely assembled by each cell type to produce distinct spatio-temporal $\mathrm{Ca}^{2+}$ signals that are selectively tailored to adjust specific cardiovascular functions (Berridge, 2003). It is, therefore, not surprising that rewiring of the $\mathrm{Ca}^{2+}$ handling machinery drives or is intimately involved in many cardiovascular diseases, including cardiac hypertrophy, chronic heart failure, ventricular and atrial arrhythmias, vascular proliferative disorders (atherosclerosis and hypertension), and diabetes (Berridge, 2003; Moccia et al., 2019).

Three of the six contributions of this Research Topic are original research articles that shed novel light on the pathogenic role of deranged $\mathrm{Ca}^{2+}$ signaling in VSMCs and cardiac myocytes and describe novel strategies to rescue cardiovascular function by dampening such aberrant elevations in $\left[\mathrm{Ca}^{2+}\right]_{i}$. Lopez et al. reported about the first description of dysregulated intracellular $\mathrm{Ca}^{2+}$ homeostasis in VSMCs deriving from an established murine model of Duchenne syndrome, known as mdx mice. These authors found that $\left[\mathrm{Ca}^{2+}\right]_{i}$ and intracellular $\mathrm{Na}^{+}$concentration $\left(\left[\mathrm{Na}^{+}\right]_{i}\right)$ were both increased in mdx VSMCs. Furthermore, they found that cyclic stretch induced a larger elevation in $\left[\mathrm{Ca}^{2+}\right]_{\mathrm{i}}$ and in $\left[\mathrm{Na}^{+}\right]_{\mathrm{i}}$ in $\mathrm{mdx}$ VSMCs as compared to wild type (WT) controls. Pharmacological analysis led the Authors to hypothesise that mechanical stimulation is sensed by G-protein coupled receptors, thereby leading to the intracellular production of diacylglycerol, followed by the activation of Transient Receptor Potential Canonical 1 (TRPC1), TRPC3, and TRPC6. Intriguingly, all these channels were upregulated and associated to higher membrane fragility in mdx VSMCs as compared to WT cells. Thus, TRPC1, TRPC3, and TRPC6 could be regarded as novel targets to prevent VSMC dysfunction in Duchenne syndrome.

Wong et al. examined the effect of topographical (microgroove-induced alignment, $\mu$ ), hormonal [triiodothyronine (T3) induction], and electrical (electrical conditioning, EC) cues on the biophysical and $\mathrm{Ca}^{2+}$-handling properties of human embryonic stem cell-derived ventricular cardiomyocytes (hESC-VCMs). High-resolution optical mapping revealed that combinatorial application of $\mu, \mathrm{T} 3$ and EC remarkably increased the conduction velocity, anisotropic ratio, 
and percentage of mature quiescent-yet-excitable preparations. This pro-maturational effect of combined $\mu$-T3-EC on hESCVCMs was associated to the upregulation of $S C N 1 B$ transcripts, and the consequent increase in the current density of the voltage-gated $\mathrm{Na}^{+}$current, while the HCN2/4 transcripts and associated funny current were downregulated. Finally, the combined $\mu$-T3-EC strategy boosted the maturation of the $\mathrm{Ca}^{2+}$ cycling machinery hESC-VCMs due to the upregulation of the transcripts encoding for transcripts encoding for sarco/endoplasmic reticulum $\mathrm{Ca}^{2+}$-ATPase (SERCA) and phospholamban transcripts. This strategy, therefore, paves the way for future investigations exploiting hESC-VCMs for cardiac disease modelling and in vitro drug screening.

Di Mauro et al. proposed a novel pharmacological approach to treat Brugada syndrome $(\mathrm{BrS})$, an inherited arrhythmogenic disease that can cause sudden death in young individuals. Approximately $12 \%$ of $\mathrm{BrS}$ cases are due loss-of-function mutations in the CACNA1C and CACNB2 genes, which, respectively, encode for the $\alpha-\left(\mathrm{Ca}_{\mathrm{V}} \alpha 1.2\right)$ and $\beta 2-\left(\mathrm{Ca}_{\mathrm{v}} \beta 2\right)$ subunits of L-type $\mathrm{Ca}^{2+}$ channels in cardiac myocytes (Napolitano and Antzelevitch, 2011). Di Mauro et al. detected two novel BrS-related $\mathrm{Ca}_{\mathrm{v}} \alpha 1.2$ mutations (T320M and Q428E) which impaired $\mathrm{Ca}_{\mathrm{v}} \beta 2$ mediated trafficking of $\mathrm{Ca}_{\mathrm{v}} \alpha 1.2$ to the plasma membrane and thereby reduced voltage-gated $\mathrm{Ca}^{2+}$ currents in a heterologous expression system. By using a mimetic peptide targeting the $\mathrm{COOH}$-terminal tail of $\mathrm{Ca}_{\mathrm{v}} \beta 2$, these Authors were able to restore $\mathrm{Ca}_{\mathrm{v}} \alpha 1.2$ trafficking to the plasma membrane, which resulted in voltage-gated $\mathrm{Ca}^{2+}$ currents similar to the WT isoform. This mimetic peptide could, therefore, represent a promising tool to treat $\mathrm{BrS}$ patients, although it remains to be probed in cardiac myocytes bearing these BrS-related $\mathrm{Ca}_{\mathrm{v}} \alpha 1.2$ mutations.

The remaining three articles of these Research Topic are reviews and perspective articles mainly dealing with the endothelial $\mathrm{Ca}^{2+}$ toolkit.

Genova et al. discussed the role played by several TRP channel isoforms in the regulation of endothelial permeability along the vascular tree. These Authors first discussed how some TRP channels, i.e., TRPC1 and TRPC4, may serve in a store-dependent manner upon their physical association with STIM1 and/or Orail to regulate the endothelial barrier function (Moccia et al., 2012). Then, they described the contribution of other endothelial TRP channels, e.g., TRPC3, TRPC4, TRP vanilloid 1 (TRPV1), TRPV4, and TRP melastatin 4 (TRPM4), in the regulation of vascular permeability. The Authors conclude that the lack of a detailed knowledge of TRP channel expression and downstream effectors in arterial/vein as compared to capillary endothelial cells is a major limitation to understand how TRP channels contribute to modulate vascular permeability.

Negri et al. described the role played by endothelial TRP channels in vascular remodelling by focusing on three main processes: angiogenesis and arteriogenesis, which are effected by vascular endothelial cells, and vasculogenesis, which is effected by endothelial colony forming cells. These Authors focused, therefore, their attention on TRPC1, TRPC3, TRPC4, TRPC5, TRPC6, TRPV1, TRPV4, TRPM2, TRPM4, TRPM7, TRP ankyrin 1 (TRPA1). Furthermore, the role of endothelial TRP channels in tumour vascularization by endothelial cells and ECFCs was also described. These processes have been mainly associated to TRPC1, TRPC3, TRPV2, TRPV4, TRPM8, and TRPA1.

The last review article of this Research Topic was by Moccia et al. who discussed the possibility to target endolysosomal two-pore channels (TPCs) to treat cardiovascular disorders in patients affected by the novel COronaVirus Disease 2019 (COVID-19). Early work showed that TPCs could be targeted to prevent Ebola virus and Middle East Respiratory Syndrome COronaVirus (MERS-CoV) infectivity (Chao et al., 2020). Therefore, the Authors speculate that TPCs also mediate severe acute respiratory syndrome coronavirus type 2 entry into cardiovascular cells, which widely express the cognate ACE2 receptor. Of note, many Food and Drug Administration-approved compounds inhibit TPCs and prevent MERS-CoV/Ebola virus infection of host cells, which may potentially expand our capability to treat COVID-19 patients with already available drugs.

\section{AUTHOR CONTRIBUTIONS}

FM drafted the manuscript and supervised the work. All authors contributed to the preparation of the manuscript and approved the submitted version.

\section{REFERENCES}

Berridge, M. J. (2003). Cardiac calcium signalling. Biochem. Soc. Trans. 31, 930-933. doi: 10.1042/bst031 0930

Chao, Y. K., Chang, S. Y., and Grimm, C. (2020). Endo-Lysosomal cation channels and infectious diseases. Rev. Physiol. Biochem. Pharmacol. doi: 10.1007/112_2020_31. [Epub ahead of print].

Eisner, D. A., Caldwell, J. L., Kistamas, K., and Trafford, A. W. (2017). Calcium and excitation-contraction coupling in the heart. Circ. Res. 121, 181-195. doi: 10.1161/CIRCRESAHA.117.31 0230

Moccia, F., Dragoni, S., Lodola, F., Bonetti, E., Bottino, C., Guerra, G., et al. (2012). Store-dependent $\mathrm{Ca}^{2+}$ entry in endothelial progenitor cells as a perspective tool to enhance cell-based therapy and adverse tumour vascularization. Curr. Med. Chem. 19, 5802-5818. doi: 10.2174/09298671280414 3240

Moccia, F., Lodola, F., Stadiotti, I., Pilato, C. A., Bellin, M., Carugo, S., et al. (2019). Calcium as a key player in arrhythmogenic cardiomyopathy: adhesion disorder or intracellular alteration? Int. J. Mol. Sci. 20:3986. doi: 10.3390/ijms20163986 
Napolitano, C., and Antzelevitch, C. (2011). Phenotypical manifestations of mutations in the genes encoding subunits of the cardiac voltage-dependent L-type calcium channel. Circ. Res. 108, 607-618. doi: 10.1161/CIRCRESAHA.110.2 24279

Smani, T., Gomez, L. J., Regodon, S., Woodard, G. E., Siegfried, G., Khatib, A. M., et al. (2018). TRP channels in angiogenesis and other endothelial functions. Front. Physiol. 9:1731. doi: 10.3389/fphys.2018. 01731

Conflict of Interest: The authors declare that the research was conducted in the absence of any commercial or financial relationships that could be construed as a potential conflict of interest.
Publisher's Note: All claims expressed in this article are solely those of the authors and do not necessarily represent those of their affiliated organizations, or those of the publisher, the editors and the reviewers. Any product that may be evaluated in this article, or claim that may be made by its manufacturer, is not guaranteed or endorsed by the publisher.

Copyright (๑) 2021 Berra-Romani, Guerra and Moccia. This is an open-access article distributed under the terms of the Creative Commons Attribution License (CC BY).

The use, distribution or reproduction in other forums is permitted, provided the original author(s) and the copyright owner(s) are credited and that the original publication in this journal is cited, in accordance with accepted academic practice. No use, distribution or reproduction is permitted which does not comply with these terms. 